\title{
DRIVER RESPONSE TO PHASE TERMINATION AT SIGNALIZED INTERSECTIONS: ARE DRIVING SIMULATOR RESULTS VALID?
}

\author{
Joshua Swake, Mafruhatul Jannat, Muhammad Islam \& David S. Hurwitz \\ Transportation Engineering, Oregon State University \\ Corvallis, Oregon, USA \\ Email: swakej@onid.orst.edu
}

\begin{abstract}
Summary: Type-II dilemma zones are the segment of roadway approaching an intersection where drivers have difficulty deciding to stop or proceed at the onset of the circular yellow indication. Signalized intersection safety is improved when dilemma zones are correctly identified and steps are taken to reduce the likelihood that vehicles are caught in such zones. This research purports that using driving simulator as a means to collect driver response data at the onset of the circular yellow indication is a valid methodology to augment our analysis of decisions and reactions made within the dilemma zone. The data obtained was compared against that from previous experiments documented in the literature and the evidence suggests that driving simulation is a valid mechanism for describing driver behavior under the given conditions.
\end{abstract}

\section{INTRODUCTION}

Numerous research efforts have focused on improving the understanding of driver behavior in response to the circular yellow (CY) indication. Rakha et al. used data from test-track experiments to gain a better understanding of driver behavior at the onset of the CY. They found that the probability of stopping varied from 100 percent at a time to stop line (TTSL) of 5.5 seconds to 9 percent at a TTSL of 1.6 seconds (2007).

Gates et al. performed field observations on over 1000 vehicles that were the first-to-stop or lastto-go at the termination of priority for that approach. The authors evaluated the effects of several variables on the decision to stop/go and reported that the factor with the most influence on driver decision making was the estimated TTSL, with the following conditions associated with a higher probability of stopping: shorter yellow interval, longer cycle lengths, vehicle type, presence of opposing roadway users, and absence of vehicles in adjacent through lanes (Gates et al, 2007).

This research aims to prove that driving simulators are valid tools for analyzing driver response to phase termination at signalized intersections. This research exploits the capabilities of a highfidelity driving simulator to measure vehicle position and speed fifteen times per second to develop a more accurate model of driver responses. Additionally, the probability-to-stop data is compared to the previous naturalistic experiments of Hurwitz et al. in 2011 and test track experiments of Rakha et al.; while the deceleration data is compared to those reported by Gates et al., and the brake-response times are compared to Gates et al., and the work of Gazis et al. in 1960, and Caird et al. in 2007. 


\section{METHODOLOGY}

\section{Driving Simulator}

The Oregon State University (OSU) driving simulator is a high-fidelity simulator, consisting of a full 2009 Ford Fusion cab mounted on top of a high performance electric pitch motion system. The pitch motion system moves +/- 4 degrees with the center of rotation around the driver's head position, allowing for accurate modeling of acceleration and braking events. Three LCOS projectors, with resolution of $1400 \times 1050$, produce a 180 x 40 degree front view and a fourth projector displays a rear image for the driver's

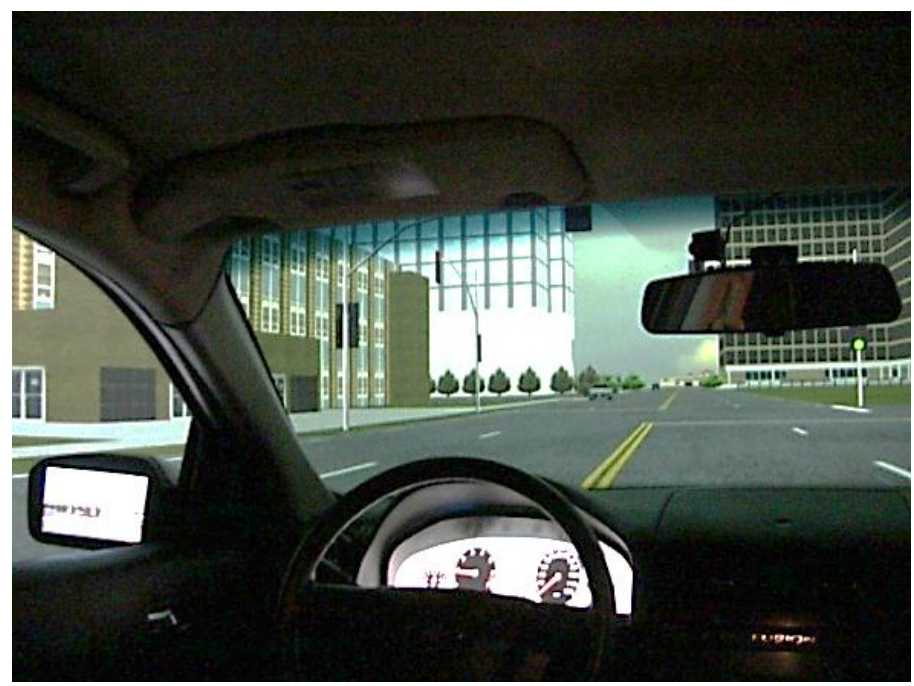
center mirror. The two side mirrors have LCD displays. The vehicle cab instruments are fully functional and include a steering control loading system to represent steering torques based on vehicle speed and steering angle. The simulator software can record performance measures such as speed, position, rake, and acceleration at a sampling rate of $60 \mathrm{~Hz}$. The simulator is pictured in Figure 1.

\section{Scenario Layout and Intersection Control}

To validate the measurements of driver response to the CY, the roadway cross-section and adjacent land use were designed to be consistent with the previous work by Rakha et al. and Hurwitz et al in 2012. In both cases, roadway cross-sections consisted of two lanes in the direction of travel, a substantial clear zone, and minimal development of adjacent land. The Rakha experiment required participants to drive along a test track at $45 \mathrm{mph}$, while the observed speed for the $85^{\text {th }}$ percentile in the Hurwitz study was $57.5 \mathrm{mph}$. With those speeds in mind, the experiment was divided into two parts: one with a posted speed of $45 \mathrm{mph}$ and one posted at 55 mph.

Within each speed condition, drivers were exposed to the CY indication at various locations on their approach to the intersection. The presentation of the CY indication was varied based on the TTSL of the driver's vehicle. Each driver was presented with the CY indication at 11 different TTSL values ranging from 1 to 6 seconds at half-second intervals. A series of 22 intersection approaches, each separated by roughly 2000 feet of roadway, were modeled forming a figureeight experimental course.

The number of participants assigned to traverse the high-speed or the low-speed portion of the track first was counterbalanced. To further eliminate confounding effects due to the order of 
exposures, each participant was exposed to a randomly generated order of TTSL triggers for the presentation of the CY indication.

A data collection sensor was placed on the approach to each intersection, tracking specified parameters from $650 \mathrm{ft}$. away from the stop line until the vehicle cleared the intersection. The parameters time, signal indication, and instantaneous speed, position, and acceleration were recorded at a rate of $15 \mathrm{~Hz}$.

\section{Texting as a Distracter}

To reduce the likelihood that participants deduced the primary research question of the study, thereby potentially altering their behavior in response, they were asked to complete several texting tasks while traversing the route. As driver's approached the horizontal curves, they were presented with a message on a billboard. Each message was a phrase or movie title in which one of the key words was left out, and the participants were asked to send a text message containing the missing word to a phone number they were given prior to experimentation. These events took place well outside the areas of interaction with the yellow indications.

\section{Participants}

A total of 30 drivers (17 male, 13 female) participated in this experiment. There was an overrepresentation of college aged students in the experiment, resulting in a relatively young subject population (average age of 24.5 years).

\section{DATA ANALYSIS AND RESULTS}

\section{Driver Decision Making}

A driver's decision to stop prior to or proceed through the intersection is the foundation for developing models to describe the Type II dilemma zone. Both speed and position are highly influential to a driver's decision; therefore driver behavior can be described in relation to the TTSL (which incorporates both factors). It was observed that all drivers proceeded through when they were 2 seconds or less from the intersection at the onset of the CY indication. This finding is consistent with the finding of Chang et al. in 1985 and Gates et al., who found that nearly all vehicles proceeded through the intersection when they were two seconds or less away at the onset of the CY. At a TTSL of 4.5 or greater, most drivers (93\%) stop before the intersection and red-light running starts to occur.

By changing the independent variable from TTSL to vehicle position, the driver's decision data can be compared to empirically observed data sets used by Rakha et al. and Hurwitz et al. 2011 Figure 2 shows the probability of stopping for all three experiments, one of which was conducted in the field, one on a test track, and one in a driving simulator. 


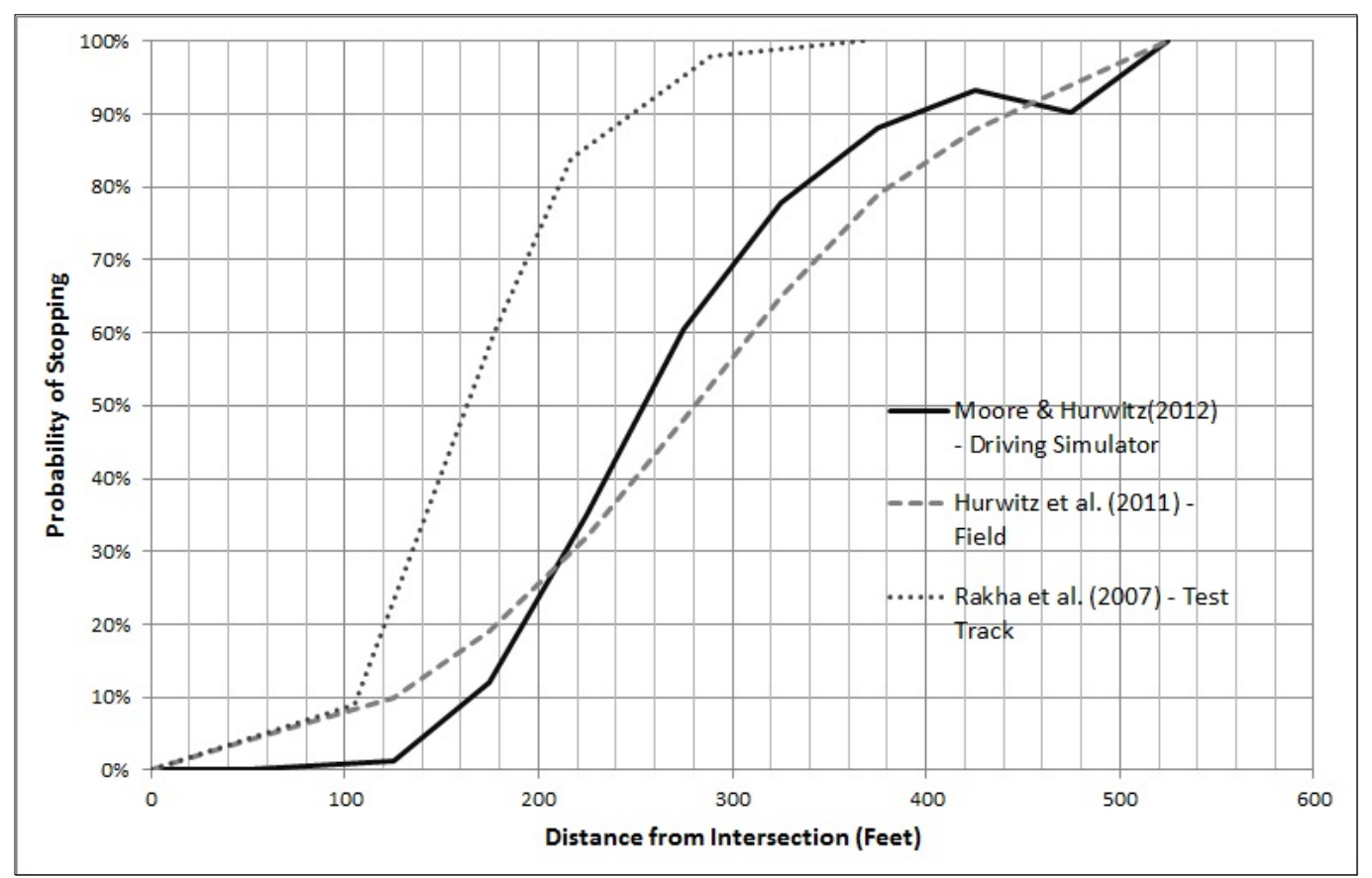

Figure 2. Probability of stopping

A two-sample Kolmogorov-Smirnov test was used to compare the three distributions. It was found that there are no statistical differences in the distributions from the previous research by Hurwitz et al. and this research $(\alpha=0.05)$, and that the distribution from Rakha et al. did not share a continuous distribution with either study ( $\alpha=0.05$ ). The curve generated for this research is similar in spread to the curve generated by Hurwitz et al. and also similar in shape with the curve generated by Rakha et al. The shift to the left associated with the Rakha et al. curve could be attributed to a lower operating speed and a reduced distance range during data collection.

\section{Deceleration Rates}

Deceleration rates are of critical importance when evaluating drivers' decisions to stop or go. The Institute of Transportation Engineers (ITE) equation for the timing of the change interval incorporates the assumption of a comfortable deceleration rate $\left(10 \mathrm{ft} / \mathrm{s}^{2}\right)$. To support the validity of using a driving simulator to evaluate driver behavior in this way, it is important that the observed deceleration rates are comparable to that threshold, as well as other studies of this nature. Average deceleration rates were calculated as the speed at initial brake application divided by the time it took to come to a complete stop. Figure 3 shows a plot of the cumulative distribution of deceleration rates for this study and several previous field studies. As shown, the deceleration rates from the simulated experiment are consistent with previous field experiments. 


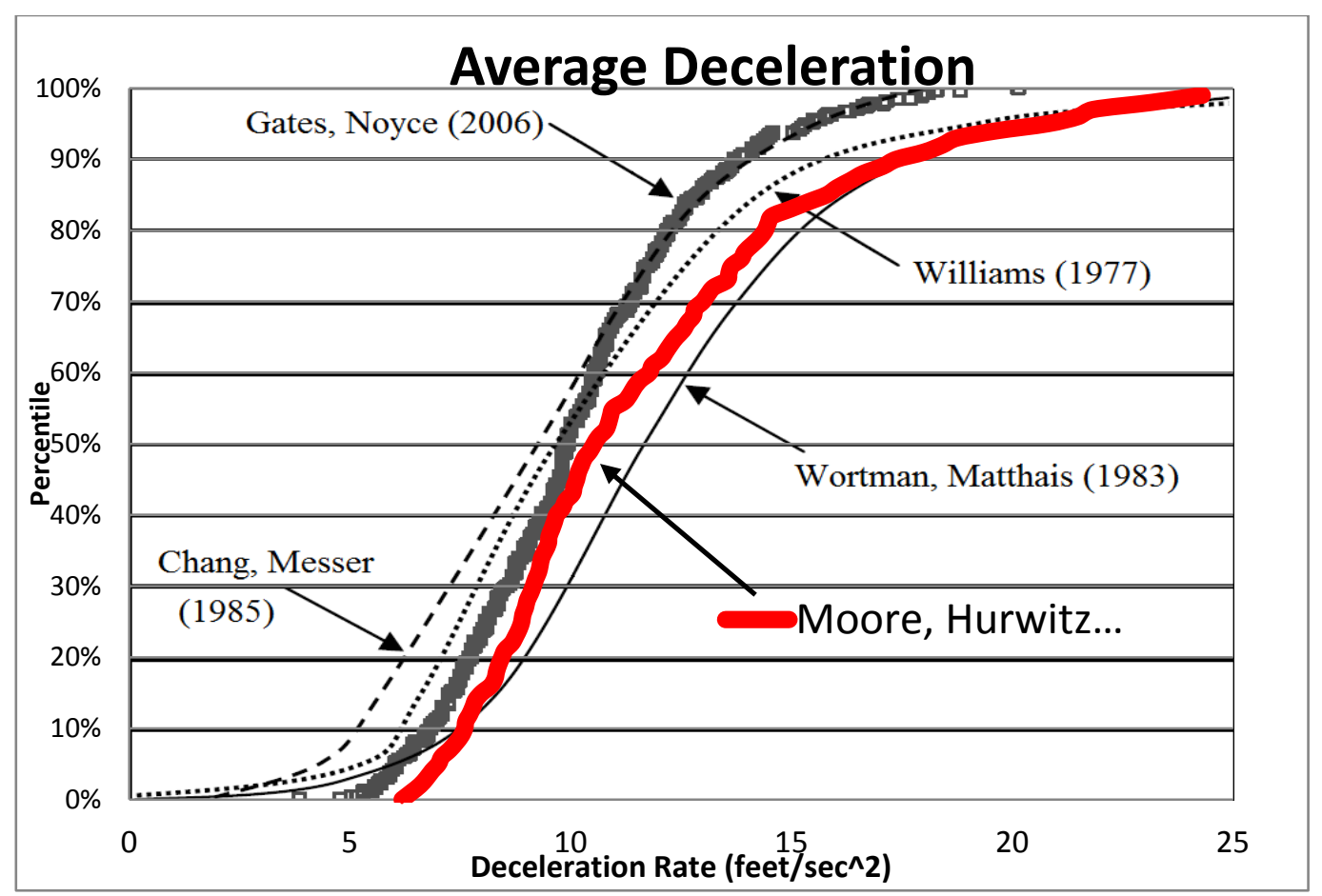

Figure 3. Average deceleration rates

Table 1 provides summary statistics comparing the deceleration rates determined from this research with three previous studies displayed in Figure 3. Deceleration rates for this experiment appear to be slightly higher than those reported by Gates et al.; however, they appear to fall within the range of values reported by other studies. Table 1 demonstrates the comparability of this data to that obtained from field observations. The 95\% confidence intervals calculated and included in Table 1 indicate no statistical difference in the mean deceleration rates from this research and the research by Gates et al. This finding provides some preliminary evidence to support the validation of deceleration rates acquired through a driving simulator in response to phase termination at a signalized intersection.

Table 1. Deceleration parameters

\begin{tabular}{|cccc|cc|ccc|}
\hline \multirow{2}{*}{ Authors } & \multirow{2}{*}{ Year } & \multirow{2}{*}{ Mean } & \multirow{2}{*}{ SD } & \multicolumn{2}{c|}{ 95\% CI } & \multicolumn{3}{c|}{ Deceleration Rate } \\
& & & & Low & High & $15 \%$ & $50 \%$ & $85 \%$ \\
\hline Moore, Hurwitz & 2012 & 11.7 & 4.0 & 3.62 & 19.78 & 8.0 & 10.5 & 15.8 \\
Gates et al. & 2006 & 10.1 & 2.8 & 4.44 & 15.76 & 7.2 & 9.9 & 12.9 \\
Chang et al. & 1985 & 9.5 & - & - & - & 5.6 & 9.2 & 13.5 \\
Wortman, Matthais & 1983 & 11.6 & - & - & - & 8.0 & 11.0 & 16.0 \\
\hline
\end{tabular}

\section{Brake Response Time}

Brake-response times are of critical importance when evaluating drivers' decisions to stop or go. The ITE equation for the timing of the change interval incorporates an assumption for a comfortable perception reaction time (PRT) of $1 \mathrm{sec}$. To further validate driving simulators as mechanisms to evaluate driver response, the observed brake-response times must compare to that 
value as well as values found in other studies of this nature. Average brake-response times were calculated as the difference between the time stamps associated with the presentation of the CY indication and that of the first significant reduction in velocity. Figure 4 plots the cumulative distribution of brakeresponse times for this study and several previous field studies. As shown on the figure below, the cumulative distribution of brake-

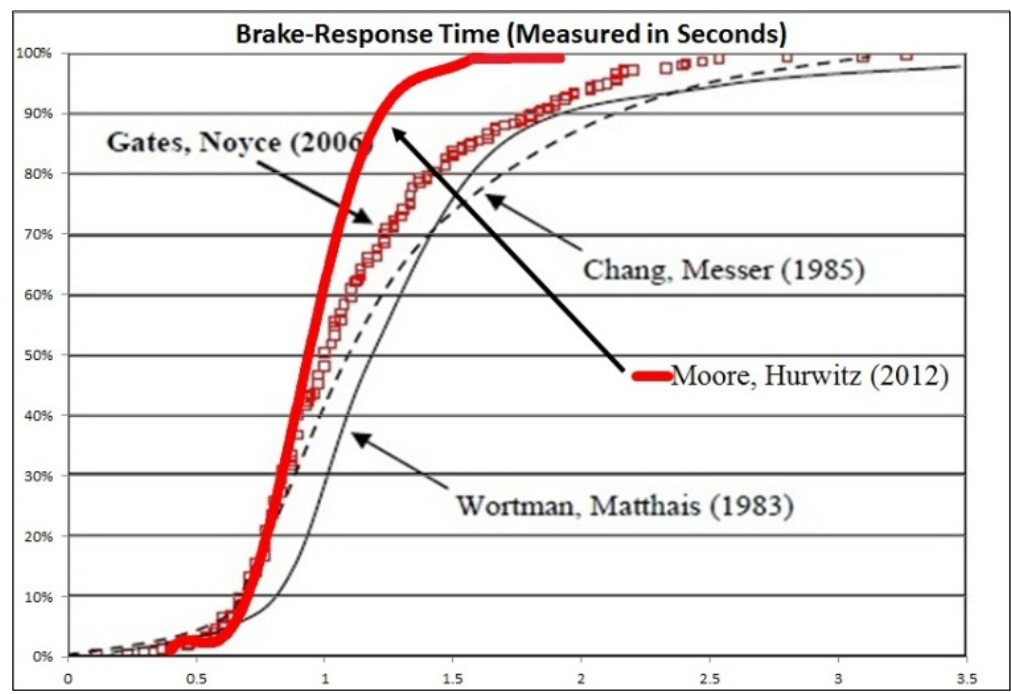
response times observed from the simulated experiment are consistent with previous field research from approximately 0 to $50 \%$.

Table 2 provides summary statistics associated with the brake-response times determined from this research as well as those displayed in Figure 4. Brake-response times for this experiment appear to be slightly lower than those reported by Gates et al., and Gazis et al.; however, they appear to fall within the range of values reported by Caird et al. Figure 4 and Table 2 demonstrate the comparability of this data to that obtained from field observations. The 95\% confidence intervals calculated and included in Table 2 indicate no statistical difference in the mean brake-response times from this research and the research by Gates et al. as well as Caird et al. and Gazis et al. This finding provides preliminary evidence to support the validation of the driving simulator for research concerning brake-response times to phase termination at signalized intersections on tangent roads.

Table 2. Brake-response parameters

\begin{tabular}{|cccc|cc|ccc|}
\hline \multirow{2}{*}{ Authors } & \multirow{2}{*}{ Year } & \multirow{2}{*}{ Mean } & SD & \multicolumn{2}{c|}{$95 \%$ CI } & \multicolumn{3}{c|}{ Brake - Reaction Time } \\
& & & & Low & High & $15 \%$ & $50 \%$ & $85 \%$ \\
\hline Moore, Hurwitz & 2012 & 0.96 & 0.22 & 0.93 & 1 & 0.7 & 0.9 & 1.2 \\
Gates et al. & 2006 & 1.1 & 0.5 & 1.05 & 1.15 & 0.7 & 1 & 1.6 \\
Caird et al. & 2005 & 0.96 & 0.27 & 0.9 & 1.02 & - & - & 1.22 \\
Rakha et al. & 2007 & 0.74 & 0.19 & 0.73 & 0.76 & - & - & 0.8 \\
Gazis et al. & 1960 & 1.1 & 0.28 & 1.08 & 1.2 & - & - & 1.5 \\
\hline
\end{tabular}

\section{CONCLUSIONS}

\section{Simulator Validation}

Driving simulation has been recognized as a safe, efficient, and effective method to evaluate driver behavior under various conditions. However, it is critically important to scope research 
questions appropriately in a driving simulator, and there is a need for extensive validation of the results obtained in laboratories of this type. As such, efforts should be made to compare results from simulator experiments with those obtained from alternative experimental mediums (surveys, test-tracks, field study, etc.).

Driver decision making, vehicle deceleration rates, and break response times are important factors when attempting to evaluate and model driver behavior in Type II dilemma zones. Driving simulator data was collected on driver decision making, rate of deceleration, and break response time and that data was compared to several previous research studies conducted in different experimental mediums. The comparison provides evidence that driver response to phase termination at traffic signals on tangent segments of roadway can be effectively evaluated and modeled in a driving simulator of a similar configuration to the one operated by the OSU Driving and Bicycling Research Lab.

\section{REFERENCES}

Caird, J.K., Chisholm, S.L., Edwards, C.J., Creaser, J.I. (2007). “The Effect of Yellow Light Onset Time on Older and Younger Drivers' Perception Response Time (PRT) and Intersection Behavior." Transportation Research Part F: Traffic Psychology and Behaviour. Vol. 10, No. 5, pp. 383-396.

Chang, M.S., C.J. Messer, C.J., and A.J. Santiago. (1985). "Timing traffic signal change intervals based on driver behavior”. Transportation Research Record: Journal of The Transportation Research Board, No. 1027, Transportation Research Board of the National Academies, Washington D.C., pp. 20-30.

Gates, T.J., D. A. Noyce and L. Larauente. (2007) “Analysis of dilemma zone driver behavior at signalized intersections.” Transportation Research Record: Journal of The Transportation Research Board, No. 2030, Transportation Research Board of the National Academies, Washington D.C., pp. 29-39.

Gazis, D., Herman, R., Maradudin, A. (1960) "The Problem of the Amber Signal in Traffic Flow.” Traffic Engineering, pp. 19-26

Hurwitz, D.S., M.A. Knodler, M.A., B. Nyquist. (2011) "Evaluation of Driver Behavior in Type II Dilemma Zones at High-Speed Signalized Intersections.” ASCE: Journal of Transportation Engineering, Vol. 137, No. 4, pp. 277-286.

Hurwitz, D.S., H. Wang., A.M. Knodler, D. Ni, and D. Moore. (2012) "Fuzzy Sets to Describe Behavior in the Dilemma Zone of High-Speed Signalized Intersections.” Transportation Research Part F: Traffic Psychology and Behavior. Vol. 15, No. 2, pp. 132-143.

Rakha, H., I. El-Shawarby, and J.R. Setti. (2007) "Characterizing driver behavior on signalized intersection approaches at the onset of a yellow-phase trigger." IEEE Transactions on Intelligent Transportation Systems, Vol. 8, No. 4, pp. 630-640.

Wortman, R. H., and J. S. Matthias. (1983) "Evaluation of Driver Behavior at Signalized Intersections.” Transportation Research Record 904, Transportation Research Board of the National Academies, Washington D.C., pp. 10-20. 